\title{
BMJ Open Integrating culturally informed approaches into the physiotherapy assessment and treatment of chronic pain: protocol for a pilot randomised controlled trial
}

Bernadette Brady, ${ }^{1,2}$ Irena Veljanova, ${ }^{3}$ Siobhan Schabrun, ${ }^{2}$ Lucinda Chipchase ${ }^{2}$

To cite: Brady B, Veljanova I, Schabrun S, et al. Integrating culturally informed approaches into the physiotherapy assessment and treatment of chronic pain: protocol for a pilot randomised controlled trial. BMJ Open 2017;7:e014449. doi:10.1136/ bmjopen-2016-014449

Received 25 September 2016 Revised 28 February 2017 Accepted 21 March 2017

\section{CrossMark}

${ }^{1}$ Departments of Pain Medicine and Physiotherapy, Liverpool Hospital, Liverpool, New South Wales, Australia

${ }^{2}$ School of Science and Health, Western Sydney University, Campbelltown, New South Wales, Australia

${ }^{3}$ School of Social Science and Psychology, Western Sydney University, Bankstown, New South Wales, Australia

Correspondence to Ms Bernadette Brady; bernadette.brady@sswahs.nsw. gov.au

\section{ABSTRACT}

Introduction There is strong evidence that

biopsychosocial approaches are efficacious in the management of chronic pain. However, implementation of these approaches in clinical practice is known not to account for the beliefs and values of culturally and linguistically diverse (CALD) patients. This limitation in translation of research contributes to the disparities in outcomes for CALD patients with chronic pain adding to the socioeconomic burden of this prevalent condition. Cultural adaptation of chronic pain assessment and management is urgently required. Thus, the aim of this pilot randomised controlled trial (RCT) is to determine the feasibility, participant acceptance with and clinical effectiveness of a culturally adapted physiotherapy assessment and treatment approach when contrasted with 'usual evidence based physiotherapy care' for three CALD communities.

Methods and analysis Using a participant-blinded and assessor-blinded randomised controlled pilot design, patients with chronic pain who self-identify as Assyrian, Mandaean or Vietnamese will be randomised to either 'culturally adapted physiotherapy assessment and treatment' or 'evidence informed usual physiotherapy care'. We will recruit 16 participants from each ethnocultural community that will give a total of 24 participants in each treatment arm. Both groups will receive physiotherapy treatment for up to 10 sessions over 3 months. Outcomes including feasibility data, acceptance with the culturally adapted intervention, functional and pain-related measures will be collected at baseline and 3 months by a blinded assessor. Analysis will be descriptive for feasibility outcomes, while measures for clinical effectiveness will be explored using independent samples t-tests and repeated measures analysis of variance. This analysis will inform sample size estimates while also allowing for identification of revisions in the protocol or intervention prior to a larger scale RCT.

Ethics and dissemination This trial has full ethical approval (HREC/16/LP00L/194). The results from this pilot RCT will be presented at scientific meetings and published in peer-reviewed journals.

Trial registration number ACTRN12616000857404
Strengths and limitations of this study

Randomised, assessor-blind and participant-blind controlled trial.

> Our study is the first to provide information on the feasibility of a culturally adapted physiotherapy assessment and treatment protocol for culturally and linguistically diverse patients with chronic pain.

- The results of this pilot trial will enable clinicians and service providers to make informed decisions regarding the feasibility of adapting physiotherapy approaches for culturally and linguistically diverse patients.

- The results will provide data for a fully powered trial.

- This is a feasibility study and as such is not adequately powered to determine treatment effectiveness.

\section{INTRODUCTION}

Chronic pain is a significant global health problem. ${ }^{1}$ With an economic cost cited between \$A34billion and US\$635billion annually, chronic pain conditions carry the second highest burden of disease globally. ${ }^{1-3}$ The high economic cost has been attributed to the complex and heterogeneous nature of conditions that sit under the banner of chronic pain. ${ }^{4}$ While many conditions may have similar biological diagnoses, wide variability in symptom behaviour and response to treatments has been observed and reported..$^{5-8}$ Understanding the factors responsible for the variability in chronic pain presentations is paramount if the personal, social and economic burden of chronic pain is to be ameliorated. ${ }^{9}$

The prevailing clinical approach to the assessment and treatment of chronic pain is based on the biopsychosocial model. ${ }^{10}$ While high-quality evidence supports the use of the biopsychosocial approach, most research into chronic pain has focused on understanding 
the biological and psychological dimensions of the model. ${ }^{911}$ Ethnocultural influences, as part of the social dimension, have been overlooked when evaluating research into interventions for chronic pain. ${ }^{12}$ As such, current pain management programmes fail to consider the relationship between ethnoculture and chronic pain and have limited applicability for contemporary multicultural societies. ${ }^{12}$

One important component of biopsychosocial management is physiotherapy. ${ }^{13}$ Our review of current chronic pain interventions, including physiotherapy, demonstrated limited effectiveness in culturally and linguistically diverse (CALD) populations. ${ }^{12}$ This may be due to variability in the explanatory frameworks held by culturally diverse communities that may not align with a biopsychosocial understanding of chronic pain. ${ }^{14-16}$ For example, in some communities, psychological influences are rejected in favour of biological explanations, while in others, spiritual, social and environmental factors are considered causative. ${ }^{14-15,17-20}$ Thus, for pain management strategies to be relevant and clinically effective in culturally diverse communities, cultural adaptation of interventions is required.

Cultural adaptation of treatment refers to the process of making therapeutic goals, language, content and processes consistent with those of the target cohort. ${ }^{21}$ Evidence from psychotherapy research suggests that when programmes are culturally adapted, treatment relevance, credibility and efficacy is enhanced. ${ }^{22}$ Therefore, the aim of this pilot randomised controlled trial (RCT) is to determine the feasibility, acceptance (measured by attendance, adherence and satisfaction) and clinical effectiveness of a culturally adapted physiotherapy assessment and treatment approach when contrasted with 'usual evidence based physiotherapy care' for three unique CALD communities. Furthermore, this pilot RCT will be used to provide data to support a sample size calculation for a fully powered trial should trends of effectiveness be present.

\section{METHODS AND ANALYSIS}

\section{Study design}

A pilot assessor and participant blinded RCT design will be used. This trial has been designed according to the CONsolidated Standards of Reporting Trials (CONSORT) statement ${ }^{23}$ and will be reported according to the Standard Protocol Items: Recommendations for Interventional Trials (SPIRIT) statement ${ }^{24}$ and the template for intervention description and replication (TIDieR) checklist ${ }^{25}$ (see online supplementary material). The trial was registered with the Australian and New Zealand Clinical Trials Registry (ACTRN12616000857404) on 30 June 2016.

\section{Participants and recruitment}

Adults (aged 18 years of age and over) with chronic non-specific musculoskeletal pain (confirmed by a clinical physiotherapy assessment) of greater than 3 months' duration and who self-identify as a member of the Vietnamese,
Assyrian or Mandaean ethnocultural community will be eligible. Prospective participants will need to consent to participate in physiotherapy treatment and be available to attend a pain management programme, if randomised to this treatment arm. Participants will be excluded if they have specific diagnoses necessitating other treatment (ie, complex regional pain syndrome, radiculopathy, wait listed for surgery or complex psychological disorders) that prevents their participation in the pain management programme. Prospective participants will be excluded if they have undergone surgery in the 3 months prior to assessment, as we cannot guarantee symptom stability. Furthermore, as participation will involve group exercise participation, participants requiring assistance for mobility or transfers (including the use of an aid other than a single point stick) will be excluded. Prospective participants will be recruited from the outpatient referral lists of two public hospital physiotherapy departments within South West Sydney Local Health District (SWSLHD) (Fairfield and Liverpool Hospitals) in Australia. The recruiting physiotherapists will screen participants for eligibility. Those meeting the inclusion criteria and consenting to participate will complete the baseline assessment and subsequent initial outcome assessment.

\section{Study procedures}

Information on study procedures is summarised in the CONSORT diagram (figure 1).

\section{Randomisation}

Sixteen consecutive participants from each community (Assyrian, Mandaean and Vietnamese) will be randomised to (1) 'culturally adapted physiotherapy assessment and treatment' or (2) 'evidence informed usual physiotherapy care'. A computer-generated sequence with a 1:1 allocation ratio for each ethnocultural community will be prepared by an independent investigator not involved in the recruitment of participants or delivery of the interventions. Labelled pieces of paper with the intervention arm will be placed in sealed opaque envelopes according to the random sequence. The envelopes will be labelled with a participant number according to their entry sequence in the study. The envelopes will be managed by a central administrative officer and kept in a secure location. This officer will be contacted once a participant is consented and will be responsible for allocating to the treatment arm and arranging subsequent physiotherapy appointments, according to the treatment allocation.

\section{Blinding}

Participants and the outcome assessor will be blinded to group allocation. Participants will be blinded to their treatment category and whether their physiotherapist has been trained in the culturally responsive protocol. The success of participant blinding will be assessed by a yes/no question: 'do you think your physiotherapist has been trained in culturally responsive treatments for chronic pain?' As both treatment arms require active treatment, it is not possible 


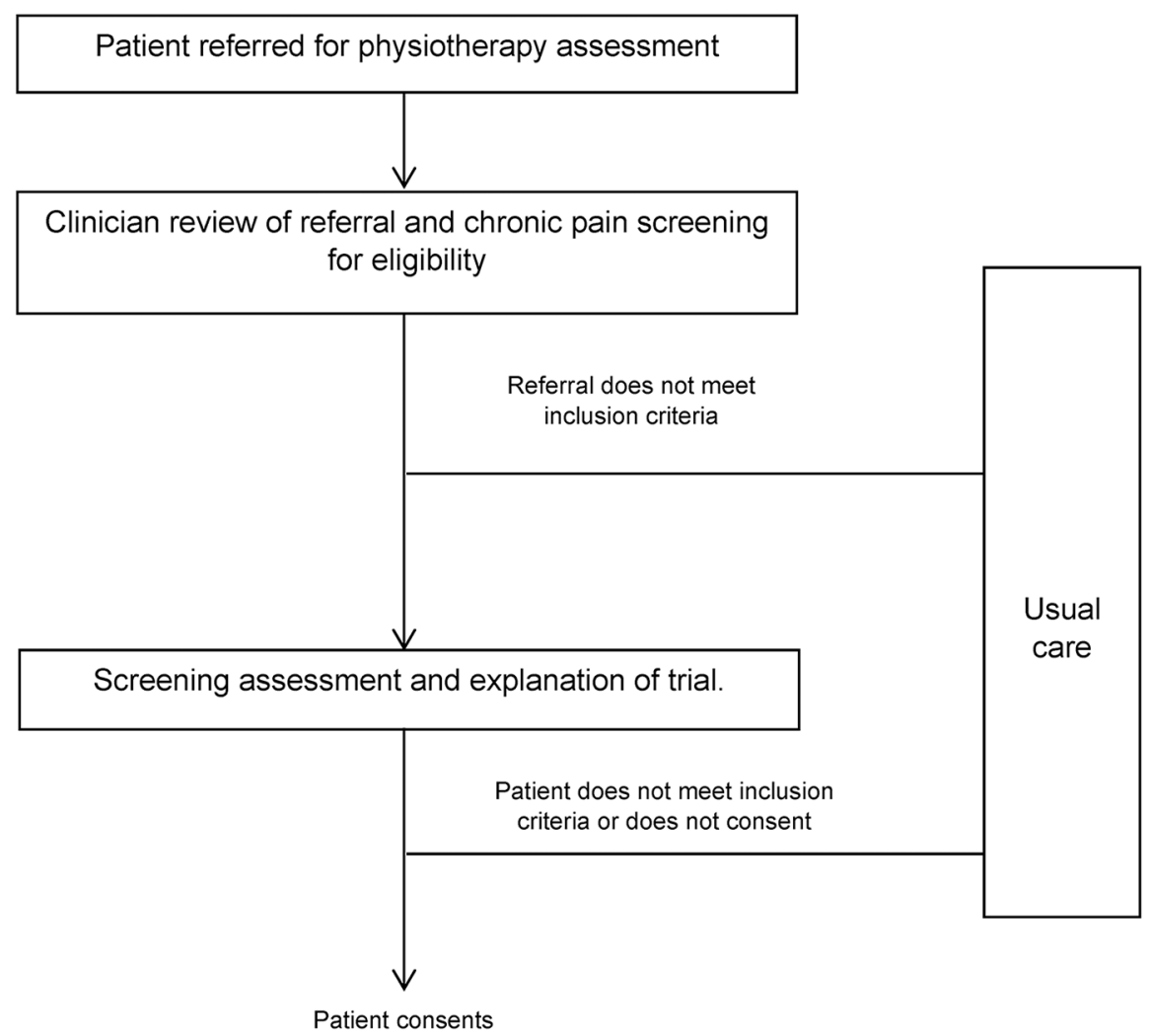

Baseline outcome measures collected and patient randomised

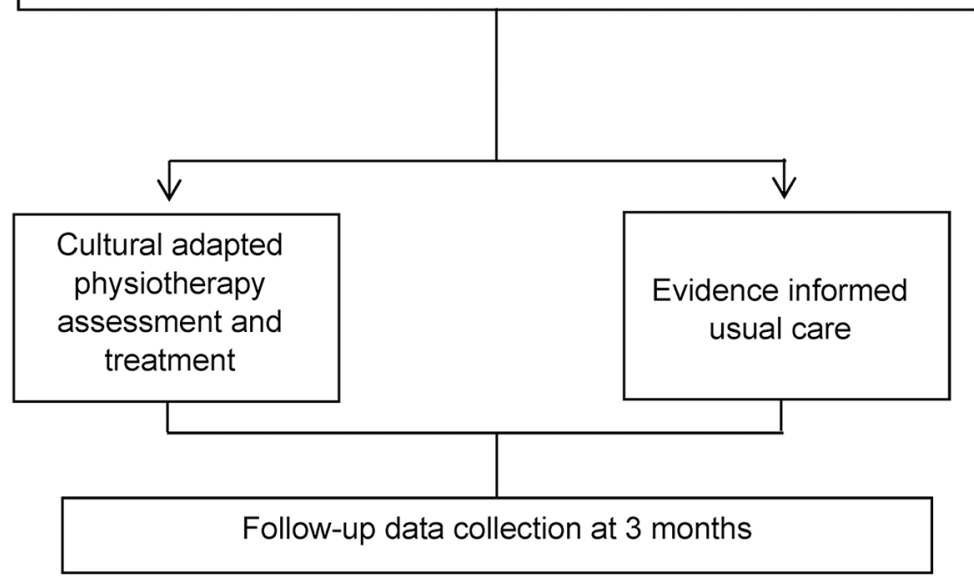

Figure 1 Trial process flow chart.

to blind the physiotherapists to treatment allocation. An investigator, blinded to the treatment allocation, will collect outcome measures at 3 months. Assessor blinding will be verified with a yes/no question: 'did you know which treatment arm the participant belonged to?'

\section{Intervention}

(1) Culturally adapted physiotherapy assessment and treatment This intervention will be delivered by a 'senior' musculoskeletal physiotherapist, ${ }^{26}$ with 11 years' clinical experience, who developed the culturally adapted pain management physiotherapy protocol. The protocol was developed following extensive qualitative enquiry, clinical expertise and review of the literature. Participants randomised to this intervention arm will attend a combination of group therapy sessions (approximately 2 hours' duration, once a week, for 6 weeks) and up to four individualised physiotherapy sessions of 30 min duration, until treatment conclusion at 3 months. Groups will be delivered in a start-stop format and limited to eight participants for each community; however, family/friends will be allowed to attend (table 1). The group sessions will be facilitated by a culturally sensitive bilingual health educator and conducted at a local community room/hall that is accessible by patients from CALD 
Table 1 Examples of culturally adapted elements

\begin{tabular}{ll}
\hline Language & Mandaean \\
& Programme content to be delivered \\
& materials translated into Arabic and \\
& reviewed by a Mandaean community \\
& member and health worker. \\
Persons & \\
& Delivered by an Arabic \\
& multicultural health worker and the \\
physiotherapist who developed the \\
culturally adapted approaches, with \\
guest speakers from the Mandaean \\
community. \\
Water, an important ethnoreligious \\
symbol for Mandaeans, used as a \\
metaphor and tool in sessions for \\
rejuvenation of the self and a means \\
of connecting with spiritual supports. \\
Culturally specific case examples \\
will be used to communicate \\
concepts such as pacing and \\
graded exposure. Spiritual relaxation \\
methods will be incorporated as \\
part of physical and emotional pain- \\
coping strategies. Culturally specific \\
exercise sessions.
\end{tabular}

\section{Assyrian}

Programme content to be delivered in Assyrian language. Materials translated into Arabic (reflective of the reading/writing language of the Iraqi Assyrian community) and reviewed by an Assyrian community member and health worker.

\section{Delivered by an Assyrian}

multicultural health worker and the physiotherapist who developed the culturally adapted approaches with guest speakers from the Assyrian community.

The giving and sharing of food will be integrated into sessions as a metaphor and means for community connectedness and support.

Culturally specific case examples will be used to communicate concepts of pacing and graded exposure. Traditional Assyrian dance will form the basis for exercise components.

\section{Vietnamese}

Programme content and materials to be delivered and translated into Vietnamese and reviewed by a Vietnamese community member and health worker.

$\begin{array}{ll}\text { Concepts } & \text { Biopsychosocial-spiritual theoretical } \\ \text { construct underpins the programme } \\ \text { content, as informed by focus group } \\ \text { findings. }\end{array}$

Goals Focused on fulfilment of traditional cultural roles and expectations. For example, goals for women will focus around ability to fulfil the role of carer and adhere to the Mandaean customs (such as prayer and food preparation customs).
Biomedical-theoretical construct underpins the adaptation of the programme content and its delivery to participants, according to the focus group findings.

Focused on fulfilment of traditional cultural roles and expectations.

For example, goals for women will focus on ability to prepare and share traditional Assyrian food with family, relatives and friends.
Delivered by a Vietnamese

multicultural health worker and the physiotherapist who developed the culturally adapted approaches with input from the Vietnamese community in traditional health practices.

Traditional Vietnamese proverbs incorporated as 'take home messages' for each session, providing a means for the sharing of advice in non-confrontational ways.

Traditional medicine components will be incorporated into painrelieving strategies. Exercise, activity and pacing will be framed with an emphasis of Am-Duong Harmony. Exercises will be categorised for participants as either Am or Duong and participants encouraged to focus on achieving balance/harmony with their programmes.

Traditional Am-Duong Medicine construct underpins the adaptation of the programme content and its delivery to participants, according to the focus group findings.

Focused on fulfilment of traditional cultural roles and expectations. For example, goals for men will focus on setting an example for the children, building self-management strategies in order to avoid burdening the family or displaying pain.

$\begin{array}{llll}\text { Methods } & \begin{array}{l}\text { Drawing on the strength of the three collectivist communities by encouraging group sharing, bringing family/ } \\ \text { friends along to the sessions and inviting community members to share their experiences and knowledge. The } \\ \text { programmes are designed to be delivered in a large community hall or group room that is located central to each } \\ \text { community. }\end{array} \\ \begin{array}{ll}\text { Recognising the social, } \\ \text { environmental, political and }\end{array} & \begin{array}{l}\text { Recognising the social, } \\ \text { environmental, political and }\end{array} & \begin{array}{l}\text { Recognising the social, environmental, } \\ \text { political and economic context }\end{array} \\ & \begin{array}{l}\text { economic context within which this } \\ \text { community experiences their pain. }\end{array} & \text { economic context within which } & \text { within which this community } \\ \text { Links and references to community } & \text { their pain. Links and references } & \text { community supports and facilitative } \\ \text { support structures such as migrant } & \text { the Assyrian Resource Centre, } & \text { programmes such as meditation } \\ \text { resource centres, community social } & \text { community social and religious } & \text { classes and public accessible exercise } \\ \text { programmes and other health } & \text { activities and other health } & \text { programmes (eg, tai chi). }\end{array}$
services.

\author{
services. \\ Recognising the social, \\ environmental, political and \\ economic context within which \\ this community experiences \\ their pain. Links and references \\ the Assyrian Resource Centre, \\ other health
}


backgrounds. Bilingual health educators will undergo two formal training sessions with the treating physiotherapist, to familiarise them with the programme content. The individual physiotherapy sessions will be delivered by the same physiotherapist and with the assistance of an interpreter as required.

The programme content is based on current evidencebased guidelines for the management of chronic pain that aim to improve patients' physical and socio-occupational functioning using cognitive behavioural approaches. ${ }^{27-29}$ Cognitive behavioural approaches, adopted by physiotherapists, are theorised to work by targeting unhelpful pain beliefs and facilitating increased confidence in movement through education, impairment focused exercise and re-engagement with usual activities despite pain. ${ }^{30}$ The success of these approaches hinge on the patient's acceptance of treatment concepts, active participation and a working therapeutic alliance between the patient and physiotherapist. These are all recognised as barriers when working with CALD patients. ${ }^{12}{ }^{31}$ To address these barriers, our programme has adapted evidence-based pain management content to reflect the beliefs, values and behaviours of the three communities of interest, using findings from our qualitative research with each ethnocultural community (table 1 ). We hypothesise that successful adaptation will ensure the mechanism of action is the same and that evidence-based pain management approaches will be applicable to these CALD communities.

Two adaptation frameworks underpinned the process of cultural adaptation. ${ }^{21}{ }^{32}$ Information gathering in the form of literature reviews, multicultural health worker consultation and focus groups was undertaken to inform the adaptation content (phase one). The findings of these focus groups are under-review elsewhere. Eight specific dimensions of physiotherapy and pain management interventions were then considered to ensure that deep and surface level adaptations were included. ${ }^{21} 33$ These included language, persons, metaphors, content, concepts, goals, methods and context ${ }^{21}$ dimensions that formed part of the manualised programme content for the three communities (phase two) (table 1). Phase three represents the current pilot phase, whereby the adapted programmes are piloted to evaluate whether the goals of the adaptation have been achieved and to identify difficulties with the programme and/or evaluation process. ${ }^{32}$ Specific examples of the adaptation elements are displayed in table 1. The programme content has been manualised and has been designed to be delivered by physiotherapists after completion of a maximum of 24 hours of further training. Training will focus on improving cultural knowledge, developing skills in cross-cultural communication, eliciting explanatory models of illness and implementing physiotherapy interventions in the context of cultural diversity. ${ }^{31}$ This dose (ie. 24hours of training) is consistent with doses reported in cultural responsiveness education programmes in the literature. ${ }^{34}$
Individual physiotherapy sessions will be tailored to suit the needs of each patient, following a culturally informed assessment. Using a pictorial tool, the physiotherapist will explore aspects of cultural beliefs and values, migration history and circumstances, degree of alignment with ethnocultural community, spiritual domains and extract the patient's explanatory model of illness. ${ }^{34}$ The remainder of the assessment, including physical examination procedures and diagnostic explanations, will be framed according to the patient's explanatory model of illness. ${ }^{35}$ Findings from our previous focus groups have highlighted that a biomedical framework is held by Assyrian communities, a biopsychosocial framework by Mandaean communities and a predominant Traditional Medicine Am-Duong framework by Vietnamese communities. The remainder of the individual sessions will be used following participation in the group programme, according to the specific needs of each individual. All participants will be given a home exercise programme, the dosage and timing of which will be determined following their culturally informed assessment.

\section{(2) Evidence-informed usual care}

Patients allocated to this treatment arm will receive treatment delivered by one of five physiotherapists who have not been trained in the culturally adapted assessment or treatment protocols. Importantly, all treating physiotherapists will be 'senior' level musculoskeletal physiotherapists $^{26}$ or have more than 4 years' clinical experience and the supervision/support of their senior and specialist musculoskeletal physiotherapy colleagues. All treating physiotherapists will attend a training session familiarising them with evidence-based guidelines for the management of chronic pain, including cognitivebehavioural therapy approaches, prior to commencing the trial. This session will incorporate concepts including explaining mechanisms of pain, goal setting, ergonomics, pacing, flare-up management, active coping strategies and exercise prescription for chronic pain.

The mode of delivery of the treatment (individual or group) is at the discretion of the therapist and what is usual practice for the specific community at each site. All participants will have access to professional health interpreting services to facilitate therapy sessions if required and will be delivered in a hospital outpatient setting. Physiotherapists will be able to use their clinical judgement to determine the specific dose and type of treatment for each patient according to their assessment. While a home exercise programme is consistent with best practice, the specifics of exercise prescription will be left to the treating physiotherapist. A maximum of 10 sessions of physiotherapy, of up to 1-hour duration (as needed), over the 3-month intervention period will be set to ensure comparability. This number is consistent with the average number of physiotherapy sessions commonly reported in clinical trials for the management of chronic pain disorders. $^{27}$ 36-41 
All treating physiotherapists will be asked to complete a treatment diary to allow adherence to the "evidence based' care or 'culturally adapted pain management physiotherapy protocol' to be established. Physiotherapist adherence to evidence-based treatment components will be reported as the percentage of core components used by the 'usual care' physiotherapist over the treatment period, calculated from therapist treatment diaries.

\section{Outcome measurements}

All personnel involved in data collection will be blinded. Study documents will be de-identified and stored in accordance with SWSLHD Human Research Ethics Committee (HREC) guidelines. Primary outcome measures include measures relevant to determine the feasibility of conducting a fully powered RCT and acceptance of the culturally adapted intervention arm. Given the complexities of research inclusive of CALD communities, challenges that may arise, as part of the clinical trial process, must be determined.

\section{Feasibility}

Feasibility data will be collected during the recruitment phase each time a new referral is assessed and will include the number of referrals screened, the number meeting the inclusion criteria and the number that agree to be randomised. The number of dropouts will be evaluated weekly until treatment conclusion and defined as the number of participants who withdraw or fail to continue with their allocated treatment. Finally, the number of participants that attend the final outcome assessment will be calculated at the 3-month assessment to ascertain the proportion of participants lost to follow-up.

\section{Acceptance (attendance, adherence and satisfaction)}

Attendance will be calculated as the proportion of the sessions attended relative to the number of sessions scheduled. Adherence to the intervention and its specific components will be calculated as the average number of home exercise sessions completed a week, relative to the number of sessions prescribed. The number of home exercise sessions per week will be calculated by review of participant logbooks and patient self-report. Both attendance and adherence will be assessed weekly from the start to treatment conclusion at 3 months. Patient satisfaction with treatment will be evaluated at the 3-month assessment using the Client Satisfaction Questionnaire (CSQ-8). ${ }^{42}$ The CSQ-8 is an eight-item instrument, available in Vietnamese and Arabic, with demonstrated reliability and excellent internal consistency for evaluating satisfaction with health services. ${ }^{42} 43$

\section{Clinical effectiveness trends}

Secondary outcome measures will include the clinical measures intended to be used in a full-scale RCT. Secondary outcome measures will be collected at baseline (pre-randomisation) and at 3-month outcome assessment.
1. The $6 \mathrm{~min}$ walk test is a reliable and valid tool that has been used to detect changes in functional performance following exercise-based interventions. It has been used in previous clinical trials and evaluated to be sensitive to change for patients with cardiac, respiratory and chronic conditions such as arthritis, fibromyalgia and chronic pain. ${ }^{44-48}$

2. The $1 \mathrm{~min}$ sit-to-stand test is a reliable and valid measure of lower body strength and endurance that has been previously used to evaluate functional performance in chronic pain, chronic obstructive pulmonary disease (COPD), renal transplant and healthy adult populations. ${ }^{49-54}$ The test represents a fundamental task performed more than 60 times/ day in a healthy adult population. ${ }^{55}$

3. The Pictorial Representation of Illness and SelfMeasure is a reliable pictorial tool to measure the burden of suffering due to an illness and has been validated for use with a range of chronic conditions including chronic pain, rheumatoid arthritis, systematic lupus erythematosus, post-traumatic stress disorder and COPD. ${ }^{56-59}$

4. The Brief Pain Inventory (Short Form) is a reliable and valid self-administered questionnaire that assesses four items of pain severity and seven items of pain interference on fundamental aspects of daily living. ${ }^{60}{ }^{61}$ It has been widely used as an outcome measure in chronic pain trials and has been validated in Arabic and Vietnamese..$^{60-66}$

5. The Depression, Anxiety and Stress Scale Short Version (DASS-21) is a 21-item self-reported questionnaire that evaluates the severity of core symptoms of depression, anxiety and stress. ${ }^{67}$ The psychometric properties of the DASS-21 have been established in a variety of chronic pain cohorts, ${ }^{68-70}$ and it has been validated for use with Arabicspeaking and Vietnamese-speaking patients. ${ }^{71} 72$

\section{Sample size calculation}

Based on previous recommendations for the sample sizes used in pilot studies and to allow for equal numbers of participants in both treatment arms, 24 participants will be recruited to each group. ${ }^{73}{ }^{74}$ According to Hertzog, ${ }^{73}$ a sample of 20 participants per group may be sufficient to generate reasonable bias-corrected estimates for medium to large effects. Therefore, to allow for equal numbers of participants in both treatment arms and the potential for dropouts, 24 participants will be recruited to each group. This will equate to 48 participants in total, of which 16 will be Assyrian, 16 Vietnamese and 16 Mandaean.

\section{Statistical analysis}

The primary quantitative analysis will focus on feasibility outcomes using descriptive analysis. The number of participants screened, participant eligibility and recruitment rates will be presented. We will also present the characteristics of participants, number of withdrawals from the 
trial for each treatment arm and the numbers/percentages of dropouts who do not attend follow-up evaluation. Acceptance of the interventions (attendance, adherence and satisfaction) will be reported using descriptive statistics with independent samples t-tests used for between group comparisons. Exploratory analysis of the treatment effect will be conducted using intention-to-treat analyses, according to last data carried forward, per group allocation. Significance levels will be set at $<0.05$ with two-tailed tests. Effect size for the secondary clinical outcome measures will be explored using repeated measures analysis of variance (ANOVA) and effect size calculated using partial $\eta^{2}$. Repeated measures ANOVA will be used with factors of intervention (usual care/ culturally adapted intervention) and time (preintervention/postintervention). If outcome data are not normally distributed, then differences will be assessed using transformed data or appropriate non-parametric tests. We will report mean differences in outcomes between arms with 95\% CIs. The size of the treatment effect will be used to determine whether it is worthwhile to conduct a fullscale RCT. All statistical analysis will be undertaken by the primary author using the Statistical Package for the Social Sciences (SPSS) Version 24, using an anonymised spreadsheet following data entry by an operator blinded to treatment allocation.

\section{ETHICS AND DISSEMINATION}

All participants will provide voluntary written informed consent. Prospective participants will be fully informed about what study participation involves and the potential benefits and risks, using an interpreter or bilingual physiotherapist where necessary. Ethics approval has been obtained from the SWSLHD HREC (HREC/16/ LPOOL/194) and Western Sydney University Human Research Ethics Committee (RH11741). Any protocol amendments will be submitted for ethical approval and communicated to the trial registry. All investigators will have access to interim analyses. The results arising from this pilot RCT will be presented at scientific meetings and published in peer-reviewed journals. There is no intention to use professional writers and authorship will be based on the International Committee of Medical Journal Editors guidelines.

\section{DISCUSSION}

This paper describes the protocol for the cultural adaptation of physiotherapy assessment and treatment of chronic pain in three CALD communities (Assyrian, Mandaean and Vietnamese). The results of the pilot trial will enable clinicians and service providers to make informed decisions regarding the feasibility of adapting physiotherapy approaches to suit the beliefs and values of culturally diverse patients. As the first trial to evaluate a culturally adapted physiotherapy assessment and treatment approach for chronic pain, this study has been primarily designed to assess the feasibility and acceptability of delivering the novel approaches. Therefore, this study is not adequately powered to determine treatment effectiveness and further studies will be necessary to demonstrate the efficacy and generalisability of the findings to physiotherapy services beyond SWSLHD and the Vietnamese, Mandaean or Assyrian communities included.

\section{TRIAL STATUS}

Recruitment started in July 2016. At the time of protocol submission this study is recruiting participants. The pilot trial is expected to be completed (including follow-up testing) by June 2017.

Contributors BB, IV, SS and LC were involved in the conception, design, writing and editing of the study protocol. All authors approved the final protocol.

Competing interests None declared.

Ethics approval South West Sydney Local Health District Human Research Ethics Committee.

Provenance and peer review Not commissioned; externally peer reviewed.

Open Access This is an Open Access article distributed in accordance with the Creative Commons Attribution Non Commercial (CC BY-NC 4.0) license, which permits others to distribute, remix, adapt, build upon this work non-commercially, and license their derivative works on different terms, provided the original work is properly cited and the use is non-commercial. See: http://creativecommons.org/ licenses/by-nc/4.0/

(c) Article author(s) (or their employer(s) unless otherwise stated in the text of the article) 2017. All rights reserved. No commercial use is permitted unless otherwise expressly granted.

\section{REFERENCES}

1. Vos T, Flaxman AD, Naghavi M, et al. Years lived with disability (YLDs) for 1160 sequelae of 289 diseases and injuries 1990-2010: a systematic analysis for the Global Burden of Disease Study 2010. Lancet 2012;380:2163-96.

2. Gaskin DJ, Richard P. The economic costs of pain in the United States. J Pain 2012;13:715-24.

3. Access Economics. The high price of pain: the economic impact of persistent pain in Australia. Sydney, Australia: Report for MBF Australia in collaboration with The University of Sydney Pain Management Research Institute, 2007. Available at. http://www. painaustralia.org.au/images/pain_australia/MBF\%20Economic\% 20lmpact.pdf.

4. Siddall PJ, Cousins MJ. Persistent pain as a disease entity: implications for clinical management. Anesth Analg 2004;99:510-20.

5. Vlaeyen JW, Morley S. Cognitive-behavioral treatments for chronic pain: what works for whom? Clin J Pain 2005;21:1-8.

6. Jensen MP, Turner JA, Romano JM, et al. Coping with chronic pain: a critical review of the literature. Pain 1991;47:249-83.

7. Fillingim RB, King CD, Ribeiro-Dasilva MC, et al. Sex, gender, and pain: a review of recent clinical and experimental findings. J Pain 2009;10:447-85.

8. Edwards CL, Fillingim RB, Keefe F. Race, ethnicity and pain. Pain 2001;94:133-7.

9. Gatchel RJ, Peng YB, Peters ML, et al. The biopsychosocial approach to chronic pain: scientific advances and future directions. Psychol Bull 2007;133:581-624.

10. Engel GL. The clinical application of the biopsychosocial model. Am J Psychiatry 1980;137:535-44.

11. Mogil JS, Simmonds K, Simmonds MJ. Pain research from 1975 to 2007: a categorical and bibliometric meta-trend analysis of every research paper published in the journal, Pain. Pain 2009;142:48-58.

12. Brady B, Veljanova I, Chipchase L. Are multidisciplinary interventions multicultural? A topical review of the pain literature as it relates to culturally diverse patient groups. Pain 2016;157:321-8.

13. Morley S, Eccleston C, Williams A. Systematic review and metaanalysis of randomized controlled trials of cognitive behaviour therapy and behaviour therapy for chronic pain in adults, excluding headache. Pain 1999;80:1-13. 
14. Scheermesser M, Bachmann S, Schämann A, et al. A qualitative study on the role of cultural background in patients' perspectives on rehabilitation. BMC Musculoskelet Disord 2012;13:5.

15. Cartwright T, Torr R. Making sense of illness: the experiences of users of complementary medicine. J Health Psychol 2005;10:559-72.

16. May CR, Rose MJ, Johnstone FC. Dealing with doubt. How patients account for non-specific chronic low back pain. J Psychosom Res 2000;49:223-5

17. Lynch E, Medin D. Explanatory models of illness: a study of withinculture variation. Cogn Psychol 2006;53:285-309.

18. Kleinman A. The illness narratives: suffering, healing, and the human condition. New York: Basic Books, 1988:1-284.

19. Garro LC. Cultural meaning, explanations of illness, and the development of comparative frameworks. Ethnology 2000;39:305.

20. Eccleston C, Williams AC, Rogers WS. Patients' and professionals understandings of the causes of chronic pain: blame, responsibility and identity protection. Soc Sci Med 1997;45:699-709.

21. Bernal G, Jiménez-Chafey MI, Domenech Rodríguez MM. Cultural adaptation of treatments: a resource for considering culture in evidence-based practice. Prof Psychol 2009;40:361-8.

22. Griner D, Smith TB. Culturally adapted mental health intervention: A meta-analytic review. Psychotherapy 2006;43:531-48.

23. Schulz KF, Altman DG, Moher D. CONSORT Group. CONSORT 2010 statement: updated guidelines for reporting parallel group randomised trials. BMJ 2010;340:c332.

24. Chan AW, Tetzlaff JM, Gøtzsche PC, et al. SPIRIT 2013 explanation and elaboration: guidance for protocols of clinical trials. $B M J$ 2013;346:e7586.

25. Hoffmann TC, Glasziou PP, Boutron I, et al. Better reporting of interventions: template for intervention description and replication (TIDieR) checklist and guide. Bmj 2014;348:g1687.

26. NSW Health. NSW health service health professionals (State) Award. Sydney, Australia. http://www.health.nsw.gov.au/careers/conditions/ Awards/health_professional.pdf (accessed 1Jul 2016).

27. Critchley DJ, Ratcliffe J, Noonan S, et al. Effectiveness and costeffectiveness of three types of physiotherapy used to reduce chronic low back pain disability: a pragmatic randomized trial with economic evaluation. Spine 2007;32:1474-81

28. Airaksinen O, Brox Jl, Cedraschi C, et al. Chapter 4. European guidelines for the management of chronic nonspecific low back pain. Eur Spine J 2006;15(Suppl 2):s192-s300.

29. Ehde DM, Dillworth TM, Turner JA. Cognitive-behavioral therapy for individuals with chronic pain: efficacy, innovations, and directions for research. Am Psychol 2014;69:153-66.

30. Hall A, Richmond H, Copsey B, et al. Physiotherapist-delivered cognitive-behavioural interventions are effective for low back pain, but can they be replicated in clinical practice? A systematic review. Disabil Rehabil 2016:1-9.

31. Brady B, Veljanova I, Chipchase L. Culturally informed practice and physiotherapy. J Physiother 2016;62:121-3.

32. Barrera M, Castro FG. A heuristic framework for the cultural adaptation of interventions. Clinical Psychology: Science and Practice 2006;13:311-6.

33. Resnicow K, Baranowski T, Ahluwalia JS, et al. Cultural sensitivity in public health: defined and demystified. Ethn Dis 1999;9:10-21.

34. Horvat L, Horey D, Romios P, et al. Cultural competence education for health professionals. The Cochrane Database Syst Rev 2014;5:CD009405.

35. Kleinman A, Eisenberg L, Good B. Culture, illness, and care: clinical lessons from anthropologic and cross-cultural research. Ann Intern Med 1978;88:251-8.

36. Kääpä EH, Frantsi K, Sarna S, et al. Multidisciplinary group rehabilitation versus individual physiotherapy for chronic nonspecific low back pain: a randomized trial. Spine 2006;31:371-6.

37. Donzelli S, Di Domenica E, Cova AM, et al. Two different techniques in the rehabilitation treatment of low back pain: a randomized controlled trial. Eura Medicophys 2006;42:205-10.

38. Frost H, Klaber Moffett JA, Moser JS, et al. Randomised controlled trial for evaluation of fitness programme for patients with chronic low back pain. BMJ 1995;310:151-4.

39. Ferreira ML, Ferreira PH, Latimer J, et al. Comparison of general exercise, motor control exercise and spinal manipulative therapy for chronic low back pain: a randomized trial. Pain 2007;131:31-7.

40. Fontaine KR, Conn L, Clauw DJ. Effects of lifestyle physical activity on perceived symptoms and physical function in adults with fibromyalgia: results of a randomized trial. Arthritis Res Ther 2010;12:R55

41. Hall A, Richmond H, Copsey B, et al. Physiotherapist-delivered cognitive-behavioural interventions are effective for low back pain, but can they be replicated in clinical practice? A systematic review. Disabil Rehabil 2016:1-9.
42. Attkisson CC, Zwick R. The client satisfaction questionnaire. Psychometric properties and correlations with service utilization and psychotherapy outcome. Eval Program Plann 1982;5:233-7.

43. Attkisson C, Greenfield T. The UCSF Client Satisfaction Scales: 1. The Client Satisfaction Questionnaie-8. Maruish M, ed. The use of psychological testing for treatment planning and outcome assessment. Mahwah, NJ: Lawrence Erlbaum Associates, 2004.

44. Peppin JF, Marcum S, Kirsh KL. The chronic pain patient and functional assessment: use of the 6-Minute Walk Test in a multidisciplinary pain clinic. Curr Med Res Opin 2014;30:361-5.

45. Pankoff B, Overend T, Lucy D, et al. Validity and responsiveness of the 6 minute walk test for people with Fibromyalgia. J Rheumatol 2000;27:2666-70.

46. Focht BC, Rejeski WJ, Ambrosius WT, et al. Exercise, self-efficacy, and mobility performance in overweight and obese older adults with knee osteoarthritis. Arthritis Rheum 2005;53:659-65.

47. O'Keeffe ST, Lye M, Donnellan C, et al. Reproducibility and responsiveness of quality of life assessment and six minute walk test in elderly heart failure patients. Heart 1998;80:377-82.

48. Hajiro T, Nishimura K, Tsukino M, et al. Analysis of clinical methods used to evaluate dyspnea in patients with chronic obstructive pulmonary disease. Am J Respir Crit Care Med 1998;158:1185-9.

49. Strassmann A, Steurer-Stey C, Lana KD, et al. Population-based reference values for the 1-min sit-to-stand test. Int J Public Health 2013;58:949-53.

50. Ozalevli S, Ozden A, Itil O, et al. Comparison of the Sit-to-Stand Test with 6 min walk test in patients with chronic obstructive pulmonary disease. Respir Med 2007;101:286-93.

51. Harding VR, Williams AC, Richardson PH, et al. The development of a battery of measures for assessing physical functioning of chronic pain patients. J Pain 1994;58:367-75.

52. Puhan MA, Siebeling L, Zoller M, et al. Simple functional performance tests and mortality in COPD. Eur Respir $J$ 2013;42:956-63.

53. Ritchie C, Trost SG, Brown W, et al. Reliability and validity of physical fitness field tests for adults aged 55 to 70 years. J Sci Med Sport 2005;8:61-70.

54. Bohannon RW, Smith J, Hull D, et al. Deficits in lower extremity muscle and gait performance among renal transplant candidates. Arch Phys Med Rehabil 1995;76:547-51.

55. Dall PM, Kerr A. Frequency of the sit to stand task: An observational study of free-living adults. Appl Ergon 2010;41:58-61.

56. Kassardjian CD, Gardner-Nix J, Dupak K, et al. Validating PRISM (Pictorial Representation of Illness and Self Measure) as a measure of suffering in chronic non-cancer pain patients. J Pain 2008;9:1135-43.

57. Büchi S, Buddeberg C, Klaghofer R, et al. Preliminary validation of PRISM (Pictorial Representation of Illness and Self Measure) - a brief method to assess suffering. Psychother Psychosom 2002;71:333-41.

58. Wittmann L, Schnyder U, Büchi S, . PRISM (Pictorial Representation of Illness and Self Measure): a new method for the assessment of suffering after trauma. J Trauma Stress 2012;25:94-7.

59. Büchi S, Villiger P, Kauer Y, et al. PRISM (Pictorial Representation of Illness and Self Measure)- a novel visual method to assess the global burden of illness in patients with systemic lupus erythematosus. Lupus 2000;9:368-73.

60. Tan G, Jensen MP, Thornby JI, et al. Validation of the brief pain inventory for chronic nonmalignant pain. J Pain 2004;5:133-7.

61. Cleeland CS, Ryan KM. Pain assessment: global use of the brief pain inventory. Ann Acad Med Singapore 1994;23:129-38.

62. Ersek M, Turner JA, Cain KC, et al. Chronic pain self-management for older adults: a randomized controlled trial [ISRCTN11899548]. BMC Geriatr 2004:4:1-11.

63. Cleeland CS. The brief pain inventory: user guide. Houston, Texas: The University of Texas 2009.

64. Keller S, Bann CM, Dodd SL, et al. Validity of the brief pain inventory for use in documenting the outcomes of patients with noncancer pain. Clin J Pain 2004;20:309-18.

65. Cleeland CS, Ladinsky JL, Serlin RC, et al. Multidimensional measurement of cancer pain: comparisons of US and Vietnamese patients. J Pain Symptom Manage 1988;3:23-7.

66. Ballout S, Noureddine S, Huijer HA, et al. Psychometric evaluation of the arabic brief pain inventory in a sample of Lebanese cancer patients. J Pain Symptom Manage 2011;42:147-54.

67. Lovibond SH, Lovibond PF. Manual for the depression anxiety stress scales. 2nd ed. Sydney, 1995.

68. Wood BM, Nicholas MK, Blyth F, et al. The utility of the short version of the Depression Anxiety Stress Scales (DASS-21) in elderly patients with persistent pain: does age make a difference? Pain Med 2010;11:1780-90.

69. Taylor R, Lovibond PF, Nicholas MK, et al. The utility of somatic items in the assessment of depression in patients with chronic pain: 
a comparison of the Zung Self-Rating Depression Scale and the Depression Anxiety Stress Scales in chronic pain and clinical and community samples. Clin J Pain 2005;21:91-100.

70. Lovibond PF, Lovibond SH. The structure of negative emotional states: comparison of the Depression Anxiety Stress Scales (DASS) with the Beck Depression and Anxiety Inventories. Behav Res Ther 1995;33:335-43.

71. Tran TD, Tran T, Fisher J. Validation of the depression anxiety stress scales (DASS) 21 as a screening instrument for depression and anxiety in a rural community-based cohort of northern vietnamese women. BMC Psychiatry 2013:13:1-7.

72. Moussa MT, Lovibond P, Laube R, et al. Psychometric properties of an arabic version of the depression anxiety stress scales (DASS). Res Soc Work Pract (Published Online First: 18 August 2016).

73. Hertzog MA. Considerations in determining sample size for pilot studies. Res Nurs Health 2008:31:180-91.

74. Viechtbauer W, Smits L, Kotz D, et al. A simple formula for the calculation of sample size in pilot studies. $J$ Clin Epidemiol 2015:68:1375-9. 